\title{
SKY BRIGHTNESS AT THE KITT PEAK NATIONAL OBSERVATORY
}

\author{
C.A. PILACHOWSKI \\ J.L. AFRICANO \\ B.D. GOODRICH \\ W.S. BINKERT \\ Kitt Peak National Observatory, Tucson, AZ U.S.A.
}

\begin{abstract}
We report on the results of a program to measure the brightness of the night sky at the Kitt Peak National Observatory during the recent minimum in the solar cycle, a time noted for the darkest nighttime skies. We have measured sky brightness using a 0.9-meter telescope with a photoelectric photometer on seven nights in 1986 and the first half of 1987. During this period, the average sky brightness in the V (yellow) band is 21.92 magnitudes per square arc-sec. This corresponds to an increase of only 6.5 percent over the natural sky illumination, as compared to an increase of over 10 percent predicted by $R$. Garstang from the presence of cities and towns in southern Arizona. The continued dark skies over Kitt Peak, despite the rapid growth of Tucson's population, can only be attributed to the effectiveness of light pollution control programs in place since 1972.
\end{abstract}

(The full text of this report will be published elsewhere, in the astronomical literature.) 